\title{
Handoff Performance Evaluation based on RSS Measurement and Threshold Distance
}

\author{
Ali H. Abdulwahhab \\ Electrical - Electronic \\ Engineering \\ Gelisim University \\ Istanbul, Turkey
}

\author{
Ali Abdulwahhab Mohammed \\ Department of Remote \\ Sensing \\ Al-Karkh University of Science \\ Baghdad, Iraq
}

\author{
Gang Su \\ School of Electronics Information \\ and Electrical Engineering \\ Changsha University \\ Changsha, China
}

\begin{abstract}
Handoff procedure enables the resumption of individuals' calls or data transform without dropping the communication during moving from on base station to new station. This article presents new handoff scheme discussing the Relative Signal Strength's (RSS) measurements, threshold distance and power of neighboring base station. The RSS threshold distance is adopted to set the handoff executing decision, and typical margin level between the reciprocal communication stations' RSS for transferring a current call into new cell. The proposed system mode has been validated by simulation experimental for the adopted parameters. The numerical simulation results show higher improvement in reducing the executing unnecessary handoffs, and decreasing the handoff's delay and the blocking calls' probability.
\end{abstract}

\section{General Terms}

Handoff

\section{Keywords}

Handoff Management, Mobile Communication, Relative Signal Strength (RSS).

\section{INTRODUCTION}

The mobile communication considerate as major part in the current life, because it has easily mobility offering the continuance servicing at any time regardless of environment. The mobile network system uses cells covering as large possible as of regions. These cells provide an opportunity to reuse frequency spectrum leading to increase the network capacity as shown in Figure 1.

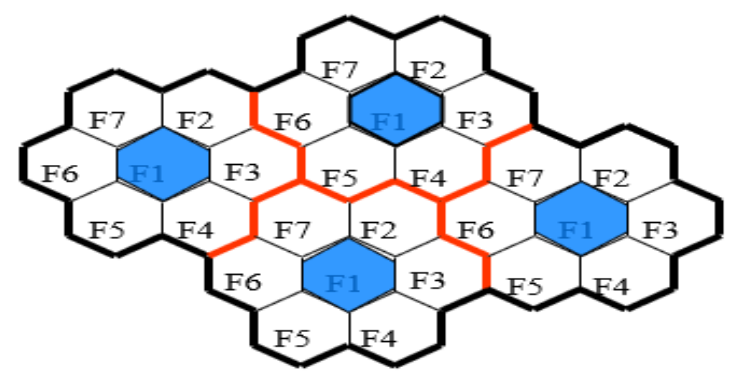

Fig 1: Region is divided into Smaller Sub-Regions Called Cells

In each cell there is a base station to control the transmitting and receiving process between the users through cell's coverage area. Usually, the call transport process from cell to other because of users moving are accompanied by blocking or dropping for the call, and this are solved by a process known the Hand-off by transferring the call data from the current base station to the new base station [1-2].

Handoff refers to a transferring's process for an ongoing call or a data connectivity from base station or one channel to another [3]. It is allowed the users to be move by providing a continuous accessing to the networks for the transmitting signals [4]. In the Next Generation Wireless System (NGWS), Handoff is classified into two types are; Vertical handoff and Horizontal handoff [5]. The vertical handoff or inter-system happens between Point of Access (PoA) supporting different network technologies, while the horizontal handoff or intersystem occurs between PoA belong to same network technologies [6]. Normally, the vertical handoff is performed during the heterogeneous cells of access systems having different in many aspects such as; data rate, frequency and bandwidth [7]. Therefore, these networks' different characteristics make the vertical handoff's implementations more challenging as compared to horizontal handoff [8].

The objective of present work is proposing a new algorithm to minimize the unnecessary handoff's number using a new handoff scheme based on Relative Signal Strength (RSS), threshold distance, and adjoining BSs power. An Additional Standard Relative Signal Strength (ASRSS) is used for analyzing the algorithm's performance based on log-normal fading environment. The algorithm's analyzing is executed according to the hand off parameters and the handoff performance criterion, that adopting on reducing both of the average handoffs' number of the setting spread parameters and mobile path, and average handoff delay. The remainder of the paper is organized as follows; in section 2 the general concept of handoff management is introduced. The system model, adopted parameters, and mathematical analysis are explained with details in Section 3. The simulation results are presented in Section 4. Finally, in Section 5 the conclusions are drawn.

\section{HANDOFF MANGEMENT}

Generally, the mobile communication consists of three components are; mobility management, location management, and handoff management [9]. Handoff Management works on enabling the network for preserving the users' connection during movement's mobile device and changing access point to the network [10]. There are three stages for the handoff management for processing as follow.

- The first stage represents the initiation from the network, user, or change the conditions of the network. It is based various criteria, such as the distance between the mobile station and surrounding base stations, the signal strengths the mobile station receives from base stations, and the bit error rates (BERs).

- The second stage refers to decision for generating a new 
connection, the network works on finding new resource to the handoff connection and implement and additional by the operating routing. There are three types of handoff decision protocols using in various cellular system which are; Network-Controlled Handoff (NCHO), Mobile Assisted Handoff (MAHO), and Mobile Controlled Handoff (MCHO) [1-11].

- The third stage is represented by controlling data flow. The data delivery from old path to the new path is maintained depending on agreed-upon service guarantees.

According to movements mobile terminal, the handoff has two types link transfer signals which are; hard-handoff and soft-handoff. [12. In hard-handoff, a break happens for the conversation or the current link before link's reestablishment with new base station (break before make connection) as shown in Figure2 - A. In the soft-handoff, at least one of the links is kept when radio links are added and removed to the mobile station" make before break" as shown in Figure2 - B [13]. The Table 1 shows the difference between the two types.

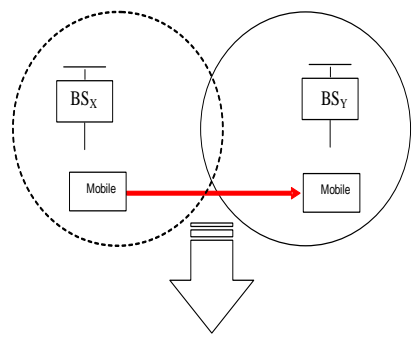

A

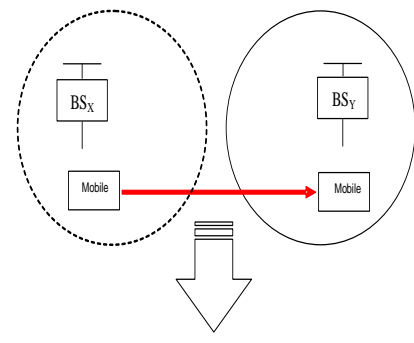

Fig 2: (A) Hard-Handoff(B)Soft-Handoff [14]

Table 1. difference between Hard and Soft handoff [14]

\begin{tabular}{|c|c|c|}
\hline Terms & Soft-handoff & Hard-handoff \\
\hline Frequency & Same freq & Different freq \\
\hline Use & $\begin{array}{c}\text { CDMA, some } \\
\text { TDMA }\end{array}$ & $\begin{array}{c}\text { TDMA and } \\
\text { FDMA }\end{array}$ \\
\hline Complexity & More complex & Less complex \\
\hline Communication & $\begin{array}{c}\text { 3-4 radio links in } \\
\text { same freq }\end{array}$ & $\begin{array}{c}\text { One BS at a } \\
\text { time }\end{array}$ \\
\hline
\end{tabular}

In generally, the efficiency evaluation of the handoff for both types based on some measurement parameters. In case of the hard-handoff the parameters are; Number of unnecessary handoffs, unsuccessful handoffs' number, expected handoffs' number, cross over point, and Expected Average Signal Strength (EASS). The soft-handoff measurement parameters are; active set updates' number, Expected number of base stations in the active set. and channel efficiency.

\section{SYSTEM MODEL}

The model comprises of two hexagonal cells as clarified in Figure3, the symbols of components and parameters clarify as follows:

- $\quad \mathrm{BS}_{\mathrm{X}}$ is the first Base Station

$\mathrm{BS}_{\mathrm{Y}}$ is the second Base Station

MD is the Mobile Device

$D_{X}$ is the BSX's radius

$D_{Y}$ is the BSY's radius

$\mathrm{D}$ is the separate distance between the BSs

- $\quad$ A is the distance between the MD's location and the $\mathrm{BS}_{\mathrm{X}}$ 's boundary.

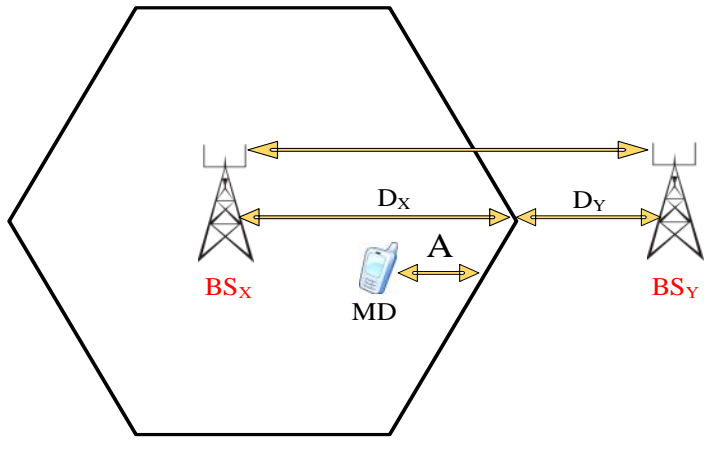

Fig 3: Adopted System Model

Once the mobile moving toward the BSX's boundary, the BSX's RSS decreases as compared to BSY's RSS, that starting increasing. The RSS's value is used to locate the threshold value. After the RSS level descends below the threshold value, the handoff is started with the adjoining BS. Two constants going to define. The first is $\mathrm{Z}$ representing the variation between the RSS of BSX and BSY, and adopts to start the handoff process. The second is threshold distance (TD).

\subsection{Relative Signal Strength (RSS)}

The RSS is measured of the presenting power of the received signal in the radio [9]. The RSS is faded rapidly during the mobile's device movement, which has similar effect for the base station receiver (BSR). On the other side, the mobile's movement in multiple paths through different and scatters terrain leads to rapid fade, and caused variation in the average signal rhythm. This fading effect is known a Shadow Fading, and follows the log-normal distribution [14]. Usually, these multipath and shadow fading outcomes many unnecessary handoffs, which are leaded to an unnecessary allocation for the channel resource or dropping the call. The handoff of mobile's calling or data connectivity is starts between the base stations after the $\mathrm{BS}_{\mathrm{X}}$ 's signal strength becomes first greater the $\mathrm{BS}_{\mathrm{Y}}$.

There is an effect known as ping-pong effect resulted due to unnecessary handoffs (increasing handoffs), which is leaded to increase the probability of forced termination due to the rapid fluctuations in the received signal strengths from both the base stations [10]. The handoff decision is determined according to the average received signal strength from the station. The MD moves with constant velocity toward the next station, and requests handing off after becoming the $\mathrm{BS}_{\mathrm{Y}}$ 's receive signal strength greater then BSx as shown in Figure4. The adopted algorithms scheme handoff for the moving in and moving out for the proposed model are presented in the Figure5, and Figure 6 respectively.

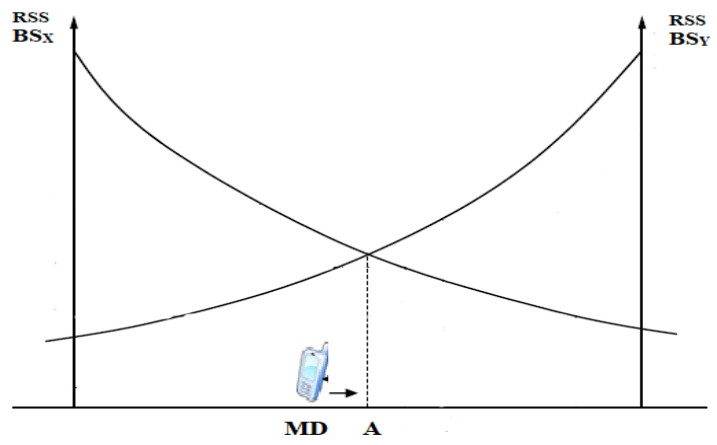

Fig 9: Handoff Vs RSS 




Fig5: Handoff Moving Out (MO) algorithm

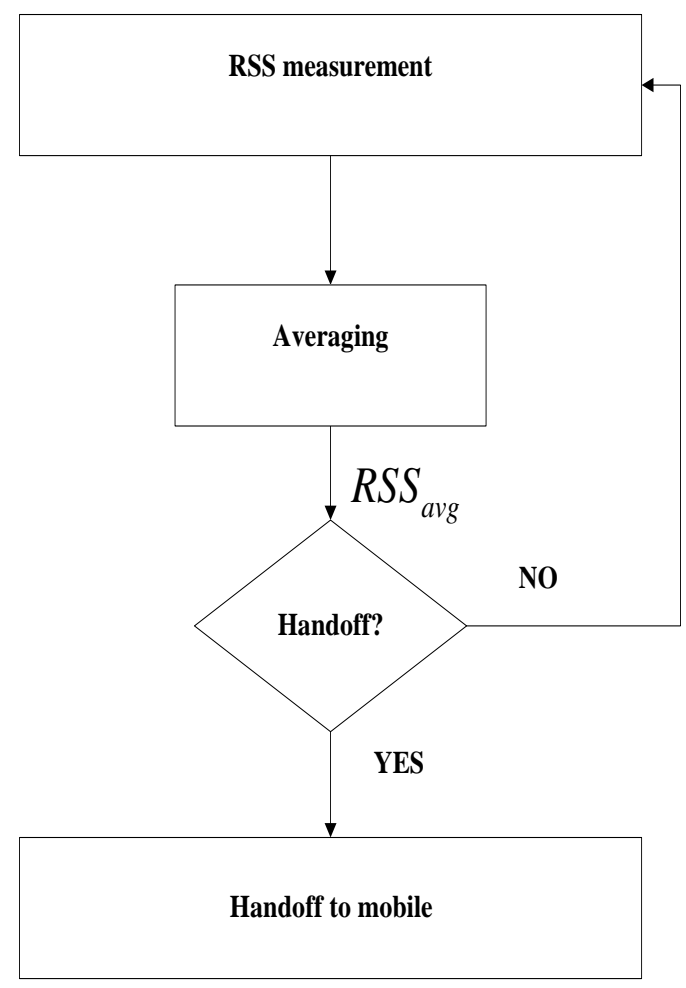

Fig6: Handoff Moving In (MI) algorithm

\subsubsection{RSS With Threshold (RSS - T)}

This method provides the overcoming the pang-pong effect, and the handoff is occurred in case the threshold is set low (T2), the mobile handoff becomes delay until the serving BS across the T2, for (T3) the mobile may be drift into new cell. the BSX's received signal strength is less than the threshold value (T1) and BSY is greater than BSX similar to performing RSS as shown in Figure7.



Fig 7: Handoff Vs RSS with T

\subsubsection{RSS with Hysteresis (RSS-H)}

The Handoff's scenario happens in this case if the BS ${ }_{Y}$ 's RSS exceeds the $\mathrm{BS}_{\mathrm{X}}$ 's RSS by hysteresis value (h) at point $\mathrm{C}$ as illustrated in Figure8. Also, the method works on preventing the ping-pong effect, but the first handoff can be unnecessary while the $\mathrm{BS}_{\mathrm{X}}$ still providing a sufficient Signal Strength (SS).

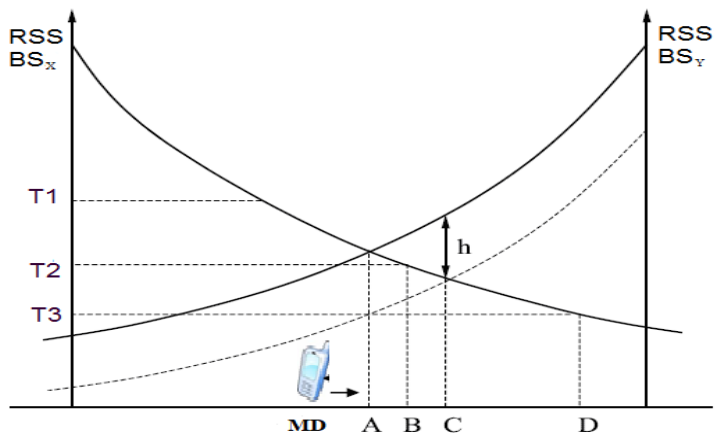

Fig 8:Handoff Vs RSS with $h$

\subsubsection{RSS with threshold and Hysteresis (RSS- TH)}

This technique uses both the threshold value and hysteresis margin for reducing the unnecessary handoff. From Figure8 above, can notice that the handoff occurs at point $\mathrm{C}$ (the threshold is T1), and at point D (the threshold is T2). In the current technique works by decreasing the threshold. Therefore, the handoff executes while the serving $\mathrm{BS}_{\mathrm{X}}$ 's SS is below the $\mathrm{T} 3$ and the $\mathrm{BS}_{\mathrm{Y}}$ 's $\mathrm{SS}$ is strong by $\mathrm{h}$.

\subsubsection{RSS with threshold Distance and Hysteresis (RSS-TDH)}

This technique works on handing off to the adjacent BS if the calculated distance from the candidate BS exceeds the serving station's distance by threshold distance under two conditions are;

- $\quad$ The adjacent BS's calculated SS exceeds the serving BS's calculated SS by h level (dB). 
- $\quad$ The serving BS's calculated SS falls below the absolute threshold value level $(\mathrm{dB})$.

\subsection{Mathematical Analysis}

The MD is moved from $\mathrm{BS}_{\mathrm{X}}$ toward $\mathrm{BS}_{\mathrm{Y}}$ with constant speed. According to the separated distance between the BSs, the received signal strength with respect to $\left(D_{X}-A\right)$ can be written as follows;

$$
\begin{aligned}
R S S_{X} & =k_{1}-k_{2} \log \left(D_{X}-A\right)+X_{X}\left(D_{X}-A\right) \\
R S S_{Y} & =k_{1}-k_{2} \log \left(D-D_{X}+A\right)+X_{Y}\left(D_{X}-A\right) \\
R S S_{Y} & =k_{1}-k_{2} \log \left(D_{Y}+A\right)+X_{Y}\left(D_{X}-A\right)
\end{aligned}
$$

Where $R S S_{X}$ and $R S S_{Y}$ are the received signal strength from $\mathrm{BS}_{\mathrm{X}}$ and $\mathrm{BS}_{\mathrm{Y}}$ respectively, $k_{1}$ and $k_{2}$ are because of the path loss, and $k_{2}=10 \mathrm{~m}, \mathrm{~m}$ represents the path loss parameter, and assumes $k_{1}$ equal to Zero and $k_{2}$ equal to 30 due to the path loss slope, which is 3 in this environment. The $X_{X}\left(D_{X}-A\right)$ and $X_{Y}\left(D_{X}-A\right)$ are the independent zero stationary Gaussian random processes modeling shadow fading. Also, the BSs' RSS could be considered to be Gaussian process average, which is expressed in equations 4 and 5 respectively.

$$
\begin{gathered}
U_{X}=k_{1}-k_{2} \log \left(D_{X}-A\right) \\
U_{Y}=k_{1}-k_{2} \log \left(D_{Y}+A\right)
\end{gathered}
$$

According to the experimental outcomes in [6], the $X_{X}(D)$ and $X_{Y}(D)$ are had an exponential correlation, which is had an auto correlation function due to the fluctuation of shadow fading, and can be given by,

$$
\begin{gathered}
E\left\{X_{X}(D) X_{Y}(D+\delta)\right\}=E\left\{X_{Y}(D) X_{Y}(D+\delta)\right\} \\
=\sigma^{\frac{2}{\delta}} \exp \left(-\frac{|\delta|}{D_{o}}\right) \\
=\sigma^{\frac{2}{\delta}} \rho^{|\delta|}(6)
\end{gathered}
$$

Where $D_{o}$ is the correlation distance using to determine how fast the factor of decaying with correlation, and $\sigma^{\frac{2}{\delta}}$ is the variance for shadow fading window, and $\rho$ is the correlation coefficient for discrete time fading. Based on the measured quantities giving the distance's functions. The MD's velocity is used for obtain the equivalent time functions using the transformation equation $D_{s}=v t_{s}$, that is known as sampling distanceadopting to calculate the impulse response. The average signals levels for the BSs using convolution are given by;

$$
\begin{aligned}
& \overline{R S S}_{X}(D)=h\left(D_{S}\right) * R S S_{X}(D) \\
& \overline{R S S}_{Y}(D)=h\left(D_{S}\right) * R S S_{Y}(D)
\end{aligned}
$$

Where the $\overline{R S S}_{X}(D)$ and $\overline{R S S}_{Y}(D)$ are the average received signals strength of BSs respectively, and the $h(D)$ is the impulse response, and calculates using the following formula;

$$
h\left(D_{s}\right)=\frac{1}{D_{a v}} \exp \left(-\frac{D_{s}}{D_{a v}}\right) D_{s} \geq 0
$$

Where, $D_{a v}$ is the smoothing window's constant distance, $D_{s}$ is the sampling distance. The average $Y_{k}$ of sequence $A_{k}$ are computed for the discrete sample by;

$$
Y_{k}=\alpha Y_{k-1}+(\alpha+1) A_{k}
$$

Generally, the handoff is executed while the $\mathrm{BS}_{\mathrm{X}}$ 's $\overline{R S S}_{X}$ is below $T d B$, and the $\mathrm{BS}_{\mathrm{Y}}$ 's $\overline{R S S}_{Y}$ is greater than $\mathrm{BS}_{\mathrm{X}}$ 's $\overline{R S S}_{X}$ by $h d B$, that is the threshold margin level to avoid the repeated handoff. The difference average received signal strength between is given by;

$$
Z(D)=\overline{R S S}_{X}(D)-\overline{R S S}_{Y}(D)
$$

For simulation studying, The Total distance $D$ is classified into small periods using $D_{s}$, that using to implement the handoff in periodically. The distance between each two neighboring sampling point (i.e., $(k-1)_{t h}$ and $\left.k_{t h}\right)$ are $D_{k-1}$ and $D_{k}$ with respect to $\mathrm{BS}_{\mathrm{X}}\left(D_{k}=k D_{s}, k=1,2 \ldots \ldots, D / D_{s}\right)$. Thus, the handoff would be occur based on the follows two cases;

- $D_{k-1}$ is the starting of the period, and represent the sampling connection point of $\mathrm{MD}$ with the $\mathrm{BS}_{\mathrm{X}}$, and the $D_{k}$ is the ending of the period, with condition $\left(\overline{R S S}_{Y}\left(D_{k}\right)>T\right.$ and $\left.Z\left(D_{k}\right)<-h\right)$ or could be,

- $\quad D_{k-1}$ is the sampling connection point of MD with the $\mathrm{BS}_{\mathrm{Y}}$, and the end at $D_{k}$ with condition ( $\overline{R S S}_{X}\left(D_{k}\right)>T$ and $Z\left(D_{k}\right)>h$ where $Z(D)=$ $\left.\overline{R S S}_{X}(D)-\overline{R S S}_{Y}(D)\right)$

\section{SIMULATION RESULTS}

The model system performance is evaluated based on the following parameters;

Distance between BSs $(D=2000 \mathrm{~m})$

Standard deviation for log-normal fading $\left(\sigma^{2}=6 d B\right)$

Correlation distance $\left(D_{o}=20 \mathrm{~m}\right)$

Average smoothing window's constant distance $\left(D_{a v}=\right.$ $30 m$ )

Sampling distance $\left(D_{s}=1 \mathrm{~m}\right)$

Parameters path loss $\left(k_{1}=0 d B, k_{2}=30 d B\right)$

Correlation coefficient $\left(\delta=\exp \left(-\frac{\delta}{20}\right)\right.$ ) resulting in $\rho=0.951$

According to the $\rho$ function, the distance is being $46 \mathrm{~m}$ during the 0.1 of correlation decays. The evaluation of handoff algorithm's performance is based on various threshold value, and the handoffs' mean number is determined according to the wide range of $h d B$. Three values are adopted for the threshold $(T d B)$ are; $-90 \mathrm{~dB},-88 \mathrm{~dB},-85 \mathrm{~dB}$. Which are used for obtaining the handoffs mean number for the MD's moving from $\mathrm{BS}_{\mathrm{X}}$ to $\mathrm{BS}_{\mathrm{Y}}$. Usually, the mean number of handoffs decrease in case the threshold value increasing. The relationship between the handoffs' mean number versus the margin level based on the adopted threshold as clarified in Figure9.

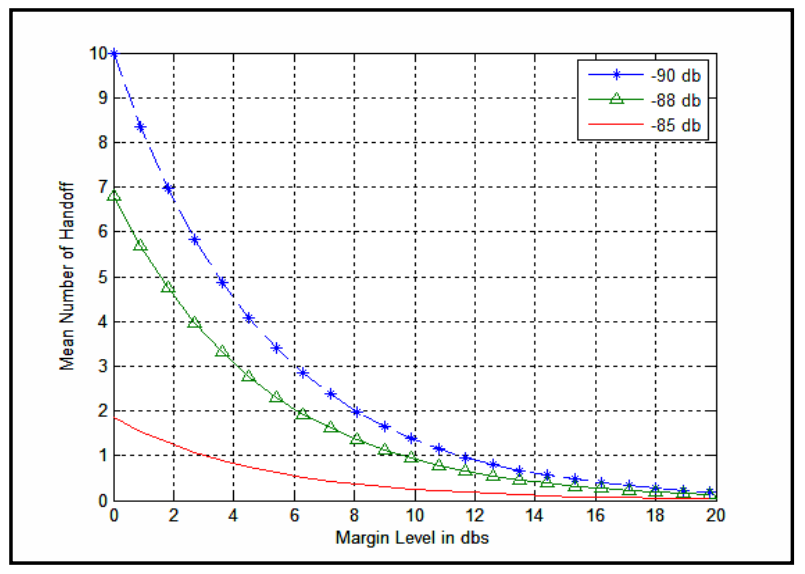

Fig9: Handoffs' mean number Vs Margin Levels. 
The curves show decreasing in handoffs' mean number while margin level increase for the three adopted threshold values, and can be noticed the handoffs' mean number improving after the $7 \mathrm{~dB}$ point and above of the margin level, which is given the best case for the handoffs' mean number in case the $T$ equal to $-80 \mathrm{~dB}$ as clarified in Table 2 .

Table 2. Handoffs' mean number Vs Margin Levels at $(T=$ $-90,-88,-85 \mathrm{dBs}$ )

\begin{tabular}{|c|c|c|c|}
\hline $\begin{array}{c}\text { Margin } \\
\text { level }\end{array}$ & \multicolumn{3}{|c|}{ Mean number of Handoff } \\
\hline & $\mathrm{T}=-90 \mathrm{~dB}$ & $\mathrm{~T}=-88 \mathrm{~dB}$ & $\mathrm{~T}=-85 \mathrm{~dB}$ \\
\hline 0 & 9.91064 & 6.72612 & 1.81548 \\
\hline 1.96262 & 6.69351 & 4.58041 & 1.24708 \\
\hline 3.97196 & 4.5179 & 3.05957 & 0.827423 \\
\hline 5.98131 & 2.99705 & 2.01491 & 0.526814 \\
\hline 7.94393 & 2.01199 & 1.35723 & 0.345322 \\
\hline 10 & 1.3244 & 0.907738 & 0.223214 \\
\hline 11.9626 & 0.875056 & 0.607198 & 0.131008 \\
\hline 13.972 & 0.574446 & 0.395875 & 0.068494 \\
\hline 15.9813 & 0.363123 & 0.244075 & 0.035742 \\
\hline 17.9439 & 0.241155 & 0.151869 & 0.00306 \\
\hline 20 & 0.14881 & 0.089286 & 0.00169 \\
\hline
\end{tabular}

The common margin level is set based on the relationship between the handoffs' mean number versus the $\mathrm{BS}_{\mathrm{X}}$ 's EASS. The handoffs' mean number decrease in case decreasing the BS $_{\mathrm{X}}$ 's EASS as presented in Figure10.



Fig 10: Handoffs' mean number Vs BS ${ }_{X}$ 's EASS.

According to the above Figure, the best case for the minimum handoffs' mean number is when $\mathrm{T}$ equal $-85 \mathrm{~dB}$. At $\mathrm{T}=-85 \mathrm{~dB}$ the $\mathrm{BS}_{\mathrm{X}}$ 's EASS has lowest values leading to decrease the unnecessary handoffs. In Figure11, the $\mathrm{BS}_{\mathrm{X}}$ 's EASS values decrease with increasing the margin levels for the adopted threshold values. The $\mathrm{BS}_{\mathrm{X}}$ 's EASS shows a decreasing above the $-95 \mathrm{~dB}$ for the $\mathrm{T}$ equal to $-90 \mathrm{~dB}$, that is gives the best handoff's mean number, and sets typical a margin level value.

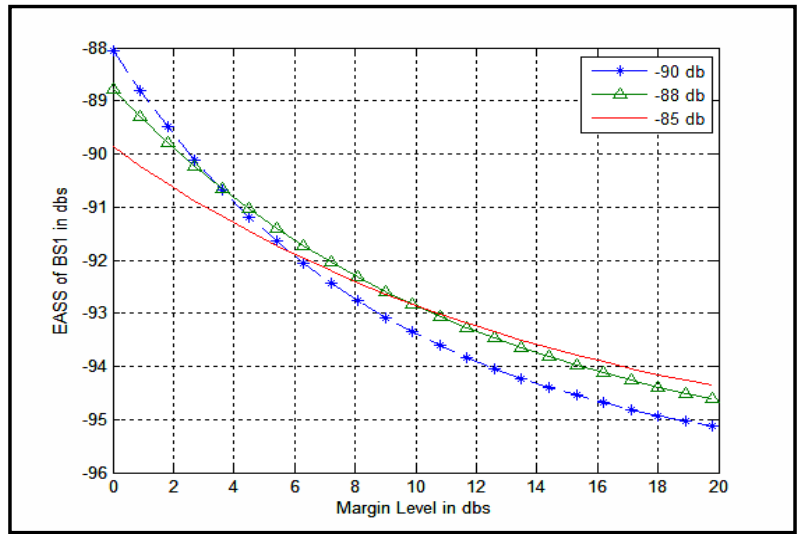

Fig11: BS $_{\mathbf{X}}$ 's EASS Vs Margin Levels.

In Figure 12, the relationship between handoffs' mean number versus threshold distance is presented. Three hysteresis levels $(0,2$, and $4 \mathrm{dBs})$ areadopted, the curves clarify the threshold distance and the hysteresis effected on the unnecessary handoffs, that is decreased if the threshold distance is increased. After $150 \mathrm{~m}$ threshold distance the handoffs' mean number becomes equal for the 2 and $4 \mathrm{dBs}$ ' hysteresis level, and becomes similar for all hysteresis levels after the $200 \mathrm{~m}$ threshold distance as listed in Table 3. comparison of the simulated handoffs' means number scheme for the proposed algorithm with the old scheme in effecting of work margin level and $\mathrm{BS}_{\mathrm{X}}$ 's EASS for threshold equal to $90 \mathrm{~dB}$ is shown in Figure 13.

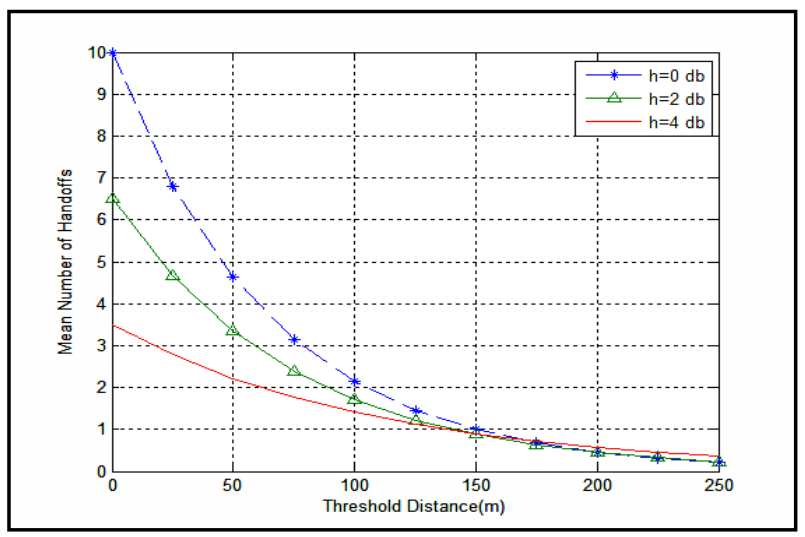

Fig 12: Handoffs' mean number Versus Threshold Distance

Table 3. Handoffs' mean number Vs Threshold distance at $(h=0,2,4 \mathrm{dBs})$

\begin{tabular}{|c|c|c|c|}
\hline $\begin{array}{c}\text { Threshold } \\
\text { Distance }\end{array}$ & \multicolumn{3}{|c|}{ Mean number of handoffs } \\
\hline & $\mathrm{h}=0 \mathrm{~dB}$ & $\mathrm{~h}=2 \mathrm{~dB}$ & $\mathrm{~h}=4 \mathrm{~dB}$ \\
\hline 0 & 10 & 6.5 & 3.5 \\
\hline 50 & 4.64706 & 3.35294 & 2.23529 \\
\hline 100 & 2.17647 & 1.73529 & 1.44118 \\
\hline 150 & 1.02941 & 0.911765 & 0.911765 \\
\hline 200 & 0.5 & 0.5 & 0.5 \\
\hline 250 & 0.235294 & 0.235294 & 0.235294 \\
\hline
\end{tabular}




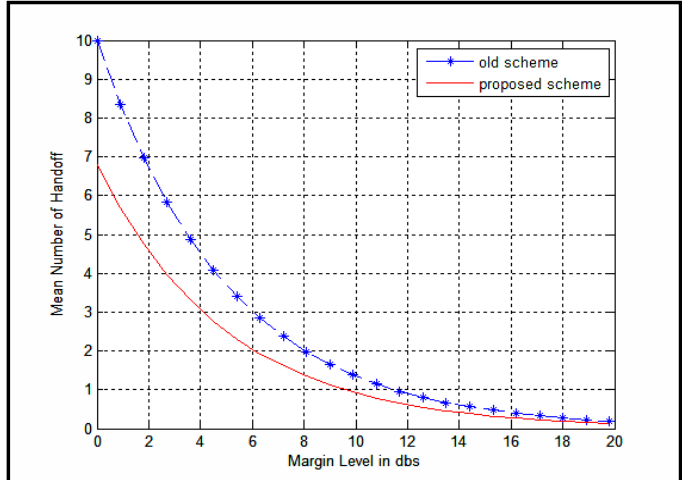

A

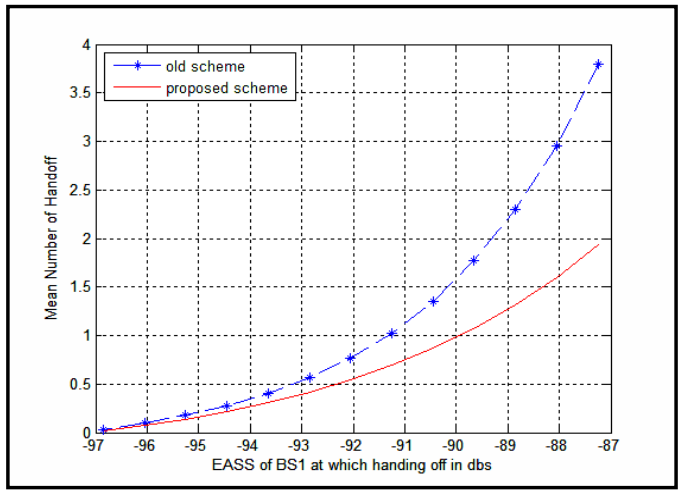

B

Fig 13:(A) Handoffs' mean number Vs Margin level (Proposed \& Old), (B) Handoffs' mean number Vs BS ${ }_{\mathrm{X}}$ 's EASS (Proposed \& Old)

\section{CONCLUSIONS}

Handoff management process using new scheme based on the relative signal strength, threshold distance and neighboring Base stations power is presented. The proposed algorithm for the system model is developed to decrease the occurring unnecessary handoffs based on the RSS measurement, the threshold margin levels, and margin level. The controlling of threshold margin level costs a delay in the handing off, regardless of reducing the handoffs' mean number. The appropriate threshold with margin level work on reducing the handoffs' mean number, and avoids early handoff in case base station serving strong signal. The handoff probability failure of priority case is reduced as compared to the non-priority case to increase the guard channels' number, which is increased the probability of new call blocking. Also, the handoff probability failure works on increasing the timer value. Also, the varying in timer value provides a wide range for the handoff scheme, which is provided an improvement in the performance through a dynamic alteration in the timer value with the traffic rhythm in the cell. The trade-off curve of handoffs' mean number versus the crossover points is used to find the optimal occurring point minimizing the handoff's delay. This leads to minimize the co-channel interface, and minimizes the expected occurring handoffs, which are worked on reducing the switching load inside network.

\section{REFERENCES}

[1] Thomas, L., Sandeep, J., Goswami, B., \& Paulose, J. 2020. A Survey on Various Handoff Methods in Mobile Ad Hoc Network Environment. In Smart Computing Paradigms: New Progresses and Challenges, pp. 27-60, Springer, Singapore.

[2] Shiri, M., \&Berangi, R.2020. Vertical handoff from WLAN to cellular network based on relay environment. Arabian Journal for Science and Engineering, Vol. 45, No.8,pp. 6259-6272.

[3] Khan, S., Iqbal, S., Qureshi, K. N., Ghafoor, K. Z., Kim, P., \& Jeon, G. 2021. Survivability of mobile and wireless communication networks by using service oriented Software Defined Network based Heterogeneous InterDomain Handoff system. Computer Communications, 175, pp. 177-185.

[4] Sreejith, S., \&Rajak, A. 2020. Study on optimization of handoff process using fuzzy logic for mobile communication. In Journal of Physics: Conference Series, Vol. 1706, No. 1, p. 012161, IOP Publishing.
[5] Parambanchary, D., \& Rao, V. M. 2020. WOA-NN: a decision algorithm for vertical handover in heterogeneous networks. Wireless Networks, Vol. 26, No. 1, pp. 165-180.

[6] Mohammed, A. A., Su, G., \&Kadhim, D. J. 2011. A new proposed handoff scheme for mobile communication systems. In Proceedings of 2011 International Conference on Computer Science and Network Technology, Vol. 4, pp. 2380-2384, IEEE.

[7] Netalkar, P. P., Maheshwari, S., \& Raychaudhuri, D. 2020. Evaluation of Network Assisted Handoffs in Heterogeneous Networks. In 2020 29th International Conference on Computer Communications and Networks (ICCCN), pp. 1-9, IEEE.

[8] Reddicherla, V. R., Rawat, U., Kumar, Y., \&Zaguia, A. 2021. Secure Vertical Handover to NEMO Using Hybrid Cryptosystem. Security and Communication Networks.

[9] Bhatt, M. C. 2017. Performance analysis of vertical handoff in Wi-Fi and WiMAX wireless networks. In 2017 2nd International Conference on Communication and Electronics Systems (ICCES), pp. 592-595, IEEE.

[10] Aljeri, N., \&Boukerche, A. 2018. Mobility and handoff management in connected vehicular networks In Proceedings of the 16th ACM International Symposium on Mobility Management and Wireless Access, pp. 82-88.

[11] Dhand, P., \& Dhillon, P. K. 2018. HANDOFF MANAGEMENT: ISSUES AND CHALLENGES. International Journal of Advanced Research in Computer Science, Vol. 9, No.1.

[12] Majumder, A., \& Nath, S. 2019. Classification of Seamless Handoff Process in Wifi Network Based on Radios. In International Conference on Computer Networks and Communication Technologies,pp. 10551065, Springer, Singapore.

[13] Neeraja, S., \& Rao, G. S. 2017. A Comparative Study on Handoff Algorithms for GSM and CDMA Cellular Networks. International Journal of Electrical \& Computer Engineering,Vol. 7, No.3, pp. 2088-8708.

[14] Ali,A, M.2012. Performance Study of a Handoff Management in Mobile Communication Systems. Master thesis, Huazhong University of Science and Technology. 